\title{
Stem Cell Research and Regenerative Medicine at King Abdullah International Medical Research Center
}

\author{
Mohamed H. Abumaree, ${ }^{1,2, *}$ Ahmed S. Al Askar, ${ }^{2,3}$ Bill Kalionis, ${ }^{4}$ Fawaz Mohamed Abomaray, ${ }^{2}$
} Dunia Jawdat, ${ }^{2,3}$ Ali H. Hajeer, ${ }^{3}$ Hana Fakhoury, ${ }^{1}$ and Mohammed A. Al Jumah ${ }^{2}$

\section{ABSTRACT}

Translation of stem cell research from bench to bedside opens up exciting new therapeutic options for patients. Although stem cell research has progressed rapidly, its clinical applications have not kept pace. We report on the establishment of a stem cell research and regenerative medicine program at King Abdullah International Medical Research Center (KAIMRC). The purpose of this unit is to coordinate advanced stem cell research and translational outcomes with the goal of treating chronic human diseases, such as cancer, diabetes, cardiovascular, neurological, immunological, and liver diseases. Our first step in achieving this goal was to integrate the stem cells and regenerative medicine unit with our umbilical cord blood bank and bone marrow registry. This organizational structure will provide different sources for stem cells for research and clinical purposes, and facilitate our stem cell research and stem cell transplantation program. We are at an early and exciting stage in our program, but we believe that our progress to the international stage will be rapid and have a significant impact.

\section{INTRODUCTION}

Saudi Arabia hosts more than 10 major research centers distributed at various universities and research institutes. These

${ }^{1}$ King Saud Bin Abdulaziz University for Health Sciences, College of Science and Health Professions, Riyadh, Kingdom of Saudi Arabia.

2 King Abdullah International Medical Research Center, Riyadh, Kingdom of Saudi Arabia.

3 King Saud Bin Abdulaziz University for Health Sciences, College of Medicine, Riyadh, Kingdom of Saudi Arabia.

${ }^{4}$ Department of Obstetrics and Gynecology and Department of Perinatal Medicine, University of Melbourne, Pregnancy Research Centre, Royal Women's Hospital, Parkville, Victoria, Australia.

*(Correspondence: abumareem@ksau-hs.edu.sa) groups conduct research in different branches of medicine, including cancer, diabetes, neurology, cardiology, ophthalmology, and reproduction. Among these groups, there are research activities into a variety of stem cell types. These research centers have established scientific backgrounds and the necessary infrastructure to support their stem cell research for the purpose of developing translational outcomes in regenerative medicine.

Stem cells are broadly classified as embryonic or adult cells. Embryonic stem cells are derived from preimplantation embryos and are pluripotent, while adult stem cells are derived from various tissues and organs and are multipotent. Regenerative medicine specialists exploit

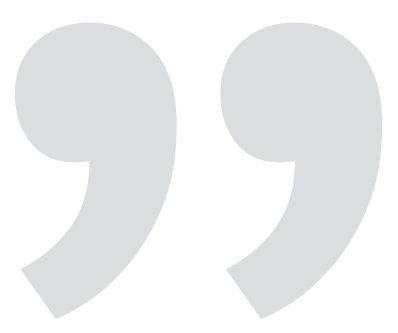

The purpose of this program is to coordinate advanced stem cell research and translational outcomes with the goal of treating chronic human diseases,

such as cancer and diabetes.

stem cells with the goal of repairing or replacing damaged cells, tissues, and organs [1] and in doing so, develop new therapies for cancer, diabetes, cardiovascular, immunological, and neurological diseases.

The leading Saudi research institutes with established stem cell research activities are found at King Saud University, King Abdulaziz University, King Khaled University, King Faisal Research Center, and King Abdullah International Medical Research Centre (KAIMRC)/King Saud bin Abdulaziz University for Health Sciences (KSAU-HS).

Stem cell research in Saudi Arabia is primarily funded by King Abdulaziz City 
for Science and Technology (KACST). In addition, each university and research institute has its own internal research funding program. Much of the research focuses on basic stem cell biology; although, several groups are performing clinical research and conducting clinical trials.

KAIMRC is a newly established and successful organization that has grown rapidly and has had impressive achievements in a short period of time. KAIMRC has several research centers in the Central (Riyadh), Eastern (Al Ahsa and Dammam), and Western (Jeddah) regions of Saudi Arabia. The broad aim of the KAIMRC is to develop the scientific research necessary to understand and explain chronic medical conditions through high-quality basic, community, and clinical research. KAIMRC has a special interest in stem cells and regenerative medicine. With the goal of integrating stem cell research activities, KAIMRC established the Stem Cells and Regenerative Medicine Unit, the Umbilical Cord Blood Bank (UCBB), and the Bone Marrow Registry. These KAIMRC facilities provide different sources for stem cells for research and clinical purposes but also act as multidisciplinary centers that attract national and international stem cell researchers. Further, these facilities form a bridge between Saudi Arabian stem research and that of the rest of the world.

KAIMRC Stem Cells and Regenerative Medicine Unit

KAIMRC Stem Cells and Regenerative Medicine Unit (KSCRMU) was established in 2009 to exploit the remarkable potential that stem cells offer in advancing human health. Our research focuses on developing new therapies for major, chronic human diseases, including cancer, diabetes, cardiovascular, neurological, immunological, and liver diseases. Our research program promotes active interaction between basic stem cell

\section{Stem Cell Research Facilities in the Kingdom of Saudi Arabia}

Saudi Arabia has been active in stem cell research for more than a decade. A 2003 fatwa, or legal decree, allows the use of embryos for therapeutic and research purposes.

Some of the major stem cell research programs in the country include:

- Stem Cell Unit, King Saud University, Riyadh

- Stem Cell Research Program, King Abdulaziz University, Jeddah

- Stem Cell Unit and Department of Clinical Biochemistry, King Khalid University, Abha

- King Faisal Specialist Hospital and Research Center, Riyadh

- Stem Cell Research and Regenerative Medicine Program, King Abdullah International Medical Research Centre (KAIMRC)/King Saud bin Abdulaziz University for Health Sciences (KSAU-HS), Riyadh

(Source: Ralston M. Stem Cell Research Around the World. July 17, 2008. Pew Research Center. www.pewforum.org/2008/07/17/stem-cell-research-around-the -world/ Last accessed 2 September 2014.

scientists and clinicians at KAIMRC and those at the King Abdulaziz Medical City. Our stem cell research program focuses on (1) isolating, characterizing, and studying the therapeutic applications of stem cells derived from human adult tissues, including placenta, umbilical cord blood (UCB) and tissue, adipose tissue, hair follicles, bone marrow, and dental pulp; (2) studying multipotent stem cell differentiation into a variety of cell types, including hepatocytes, neurons, and insulin-secreting cells; (3) developing bioengineered scaffolds to grow new tissues from stem cells; (4) investigating the interaction between stem cells and cancer cells; (5) determining the feasibility of human stem cells in modulating the immunopathogenic processes that underlie multiple sclerosis; (6) studying the role of stem cells in hair repair; (7) exploring the therapeutic potential of stem cells in liver fibrosis and in atherosclerosis; and (8) identifying new stem cell markers with potential therapeutic application, such as antitumor, antifibrotic, and antiatherosclerotic markers.

KSCRMU is equipped with state-of-theart technology, which will speed up the development of stem-cell-based therapies. We have world-class facilities for carrying out cell culture, flow cytometry, microscopy, histology, molecular biology, and animal studies. These facilities allow our scientists to conduct research that spans the spectrum from basic to advanced, specialized, clinical research.

KSCRMU, with support funding from KAIMRC, will construct a multiuse stem cell cGMP facility (SCGMPF). This will be a fully accredited facility designed for the large-scale manufacture of stem cells, which will speed up the early phases of clinical trials. The SCGMPF will be an important resource for the KAIMRC and will also serve as a local resource for stem cell scientists and clinicians. KSCRMU will also offer a range of teaching opportunities for junior and senior scientists who are interested in developing a career in stem cells and regenerative medicine.

\section{KAIMRC cord blood bank}

In 1978, hematopoietic stem cells were discovered in the UCB [2] and, since the late 1980 s, scientists have examined the potential of using UCB as an alternative to bone marrow in transplantation. The first hematopoietic stem cell transplantation was reported in 1989 [3]. Since then, CB 
transplantations have become an increasingly feasible source for treating immune system, blood-related disorders and genetic diseases. Moreover, UCBBs have been established worldwide for the collection and cryopreservation of UCB for allogeneic hematopoietic stem cell transplantation.

Currently, hematopoietic stem cells from UCB are regularly used in allogeneic transplantation. Worldwide, at present, there are around 600,000 units of stored $\mathrm{UCB}$, and at least 20,000 units have been used in transplantations to treat patients with severe hematological diseases, such as leukemia, lymphoma, and sickle cell disease [4].

Recent research confirms that $\mathrm{CB}$ is a valuable alternative source of hematopoietic stem cells for transplantation to treat hematologic malignancies, metabolic diseases, and immune deficiencies [5-7]. The advantages of using CB include rapid availability, particularly for ethnic minorities; isolation by noninvasive procedure with minimal risk factors for the donor; low risk of graftversus-host disease; and minimal risk of transmissible infection [8-10]. The main drawbacks of using cord blood are the small amount of blood remaining in the cord or placenta after delivery, which affects the total yield of hematopoietic stem cells, and the significant delays in the rates and kinetics of neutrophil and platelet engraftment following UCB transplants.

Due to the success of CB transplantation, Cord Blood Banks (CBBs) are now well established in the United States, Europe, and Australia [11]. In contrast, the establishment of $\mathrm{CBBs}$ in the Arab world is still relatively new. As well, the full potential of cord blood therapy in the Arab world has not yet been explored. Recently, several Arab countries have advanced their scientific and medical institutes, with the goal of combining $\mathrm{CB}$ banking with the utilization of $\mathrm{CB}$ stem cells for a variety of regenerative medicine applications. Nevertheless, there is still scope for much improvement with regard to funding, policies, public awareness, and scientific research in the Arab world.

Arab countries that have started to invest in $\mathrm{CB}$ banking and $\mathrm{CB}$ transplantation include Jordan, Saudi Arabia, Qatar, Egypt, and the United Arab Emirates (UAE). In the Arab world, there are many factors that provide a strong incentive for developing successful $\mathrm{CBB}$ and $\mathrm{CB}$ transplantation programs. These include high fertility rates, supportive religious leaders, a high incidence of diseases that are treatable by cord blood transplants, the scarcity of Arab bone marrow and CB units in international registries, and lower costs associated with regional storage of units. These factors were important drivers for the establishment of the UCBB at KAIMRC.

KAIMRC-UCBB is a nonprofit public bank that provides hematological stem cells for patients who are in need or for research. KAIMRC UCB bank was established in 2009. The aim of KAIMRC$\mathrm{CBB}$ is to maximize the $\mathrm{CB}$ inventory in terms of quantity and quality of stored hematopoietic stem cell units for patient care and research purposes in the most efficient, cost effective, and ethical manner possible. Although KAIMRCUCBB is a public bank, its main goal is patient care and, therefore, KAIMRC accepts intended donations for families with medical history that require $\mathrm{CB}$ stem cell donation from a family member as indicated and requested by a transplant physician.

The activities of the bank are performed within a quality system compliant with the international standards, including FACT standards and national legislations; these include donor management and recruitment, consent process, donor selection, donor screening, cord blood collection, cord blood processing and cryopreservation, and cord blood storing, listing, searching, selection, reservation, releasing, and distribution.

KAIMRC-UCBB collects the UCB samples from pregnant women who meet the selection criteria. This includes donors who must be over 34 weeks of gestation, no inherited diseases, negative for transmissible diseases, and no sign of any infection. In addition, the newborn must have no congenital abnormalities and hemoglobinopathies. The collected CB units are then screened for eligibility for processing and storage. This includes physical check for label discrepancy and ID verification, presence of clots and/or clumps, and collection bag integrity, collected volume, and number of total nucleated cells (TNCs). CBUs with TNCs $>80 \times 10^{7} / \mu \mathrm{L}$ will be processed and stored for potential clinical use. Units with TNC $50-80 \times 10^{7} / \mu \mathrm{L}$ will be processed and stored for research and quality control purposes. Other units that are not eligible for further processing and storage are discarded.

All processed units will be examined for transmissible disease markers, hemoglobinopathies, blood grouping, and human leukocyte antigen (HLA) typing. In addition to sterility testing, the total number of $\mathrm{CD}_{4} 5^{+}$and CD $34^{+}$cells will be counted and cell viability will also be measured before and after CB processing to ensure the recovery and safety of the unit. When a matched patient is found, a $\mathrm{CB}$ unit will be prepared for patient infusion. So far, we have collected 1,383 units, so we are still in the establishment phase. The first cord blood unit was stored in June 2010. We have used one unit for unrelated cord blood transplantation. It was conducted in June 2013 in a child with juvenile myelomonocytic leukemia. The cord blood unit was engrafted successfully and the child is currently healthy with no signs of graft-versus-host disease. To complement the clinical transplantation activities, several research projects employ cord blood units to investigate many important issues in the field of hematopoietic stem cell transplantation. At present KAIMRC cord blood bank is preparing for FACT accreditation and to join international registries. In the near future, KAIMRC cord blood bank is also planning to store cord tissue with the $\mathrm{CB}$ units as a source for mesenchymal stem cells. In addition, we will store units for potential autologous transplants for 
children with cerebral palsy and hypoxic ischemic encyphalopathy. Finally, at KAIMRC, we have extensive education programs to increase awareness of the medical community, and the general public, of the benefits of cord blood banking, and the value of cord blood donation.

\section{Bone marrow registry}

In Saudi Arabia, the chance of finding a matching sibling is $\sim 40 \%$ in children and $70 \%$ in adults, which is considerably higher compared with Western countries, where it is difficult to find a related donor [12]. The first bone marrow unrelated registry was launched in the 70 s in the United Kingdom, and currently there are 65 donor registries in 44 countries (with $>17,000,000$ donors) [13]. KAIMRC
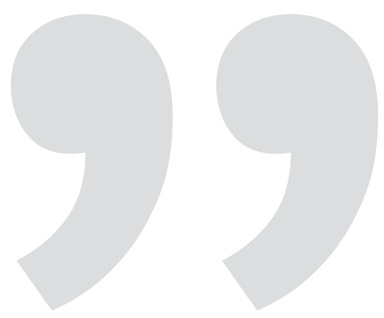

\section{KAIMRC established the first Stem Cell Donor Registry within the Arab world, in Riyadh, Saudi Arabia in 2011.}

proudly established the first Stem Cell Donor Registry (SSCDR) within the Arab world, in Riyadh in 2011.

The goal of the SSCDR is to provide stem cells from an HLA-identical, unrelated donor to both national and international patients, but priority is given to finding donors for Saudi patients with various hematological and immunodeficiency disorders. National collaborations have commenced with different cities in Saudi Arabia. Moreover, we have established international collaborations with BMDW
(Bone Marrow Donors Worldwide) and with 65 stem cell donor registries worldwide. Globally, this has provided us access to 23 million donors.

Our plan is to collect 100,000 donors over the next 5 years. All donors will be HLA typed using sequence-based typing. Thus far we have recruited 5,400 donors, of whom 2,109 were HLA typed. Clearly, we are still in the establishment phase, but already we have identified three matched donors: two local patients and one international patient.

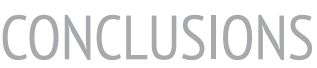

Stem cells have high potential in regenerative medicine to treat chronic diseases. Therefore, KAIMRC has invested heavily in providing, and integrating, the necessary facilities and platform technologies. Equally important is that the KAIMRC has established local, national, and international networks of scientists, clinician researchers, and collaborators to carry out the work. Already we are seeing the benefit of this investment; however, much work remains to be carried out in the laboratory and in the clinic to achieve our goal of developing stem-cell-based therapies to treat disease.

\section{Author Disclosure}

\section{Statement}

The authors declare no potential conflicts of interest.

\section{REFERENCES}

1. Mason C and P Dunnill. (2008). A brief definition of regenerative medicine. Regen Med 3:1-5.

2. Prindull G, B Prindull and N Meulen. (1978). Haematopoietic stem cells (CFUc) in human cord blood. Acta Paediatr Scand 67:413-416.

3. Gluckman E, HA Broxmeyer, AD Auerbach, HS Friedman, GW Douglas,
A Devergie, et al. (1989). Hematopoietic reconstitution in a patient with Fanconi's anemia by means of umbilical-cord blood from an HLA-identical sibling. N Engl J Med 321:1174-1178.

4. Gluckman E, A Ruggeri, F Volt, R Cunha, K Boudjedir and V Rocha. (2011). Milestones in umbilical cord blood transplantation. Br J Haematol 154:441-447.

5. Fernandes JF, V Rocha, M Labopin, B Neven, D Moshous, AR Gennery, et al. (2012). Transplantation in patients with SCID: mismatched related stem cells or unrelated cord blood? Blood 119:29492955.

6. Kamani NR, MC Walters, S Carter, V Aquino, JA Brochstein, S Chaudhury, et al. (2012). Unrelated donor cord blood transplantation for children with severe sickle cell disease: results of one cohort from the phase II study from the Blood and Marrow Transplant Clinical Trials Network (BMT CTN). Biol Blood Marrow Transplant 18:12651272.

7. Kurtzberg J, VK Prasad, SL Carter, JE Wagner, LA Baxter-Lowe, D Wall, et al. (2008). Results of the Cord Blood Transplantation Study (COBLT): clinical outcomes of unrelated donor umbilical cord blood transplantation in pediatric patients with hematologic malignancies. Blood 112:4318-4327.

8. Barker JN, TP Krepski, TE DeFor, SM Davies, JE Wagner and DJ Weisdorf. (2002). Searching for unrelated donor hematopoietic stem cells: availability and speed of umbilical cord blood versus bone marrow. Biol Blood Marrow Transplant 8:257-260.

9. Eapen M, P Rubinstein, MJ Zhang, C Stevens, J Kurtzberg, A Scaradavou, et al. (2007). Outcomes of transplantation of unrelated donor umbilical cord blood and bone marrow in children with acute leukaemia: a comparison study. Lancet 369:1947-1954.

10. Behzad-Behbahani A, R Pouransari, SZ Tabei, MS Rahiminejad, M Robati, R Yaghobi, et al. (2005). Risk of viral transmission via bone marrow progenitor cells versus umbilical cord blood hematopoietic stem cells in bone marrow transplantation. Transplant Proc 37:32113212. 
11. Barker JN, CE Byam, NA Kernan, SS Lee, RM Hawke, KA Doshi, et al. (2010). Availability of cord blood extends allogeneic hematopoietic stem cell transplant access to racial and ethnic minorities. Biol Blood Marrow Transplant 16:1541-1548.

12. Jawdat DM, S Al Saleh, P Sutton, $\mathrm{H} \mathrm{Al}$ Anazi, A Shubaili, H Tamim, et al. (2009). Chances of Finding an HLA-Matched
Sibling: The Saudi Experience. Biol Blood Marrow Transplant 15:1342-1344.

13. Cleaver SA. (1993). The Anthony Nolan Research Centre. Bone Marrow Transplant 11 Suppl 1:38-40. 